\title{
Building Large-Scale Programmable Photonic Circuits Using Silicon Photonic MEMS
}

\author{
Wim Bogaerts ${ }^{\mathrm{a}}$, Pierre Edinger ${ }^{\mathrm{c}}$, Alain Yuji Takabayashi ${ }^{\mathrm{b}}$, Iman Zand ${ }^{\mathrm{a}}$, Xiangfeng \\ Chen $^{\mathrm{a}}$, Xiaojing Wang ${ }^{\mathrm{c}}$, Hamed Sattari ${ }^{\mathrm{b}}$, Peter Verheyen ${ }^{\mathrm{g}}$, Moises A. Jezzini ${ }^{\mathrm{d}}$, \\ Giuseppe Talli ${ }^{\mathrm{d}}$, Saurav Kumar ${ }^{\mathrm{f}}$, Marco Garcia Porcele ${ }^{\mathrm{e}}$, Antonio Ribeiro ${ }^{\mathrm{a}}$, Gaehun \\ $\mathbf{J o}^{\mathbf{c}}$, Niels Quack ${ }^{\mathrm{b}}$, Kristinn B. Gylfason ${ }^{\mathrm{c}}$, Frank Niklaus ${ }^{\mathrm{c}}$, Umar Khan ${ }^{\mathrm{a}}$ \\ ${ }^{a}$ Ghent University - IMEC, Photonics Research Group, Department of Information Technology, Belgium \\ ${ }^{b}$ École Polytechnique Fédérale de Lausanne (EPFL), 1015 Lausanne, Switzerland \\ ${ }^{c}$ KTH Royal Institute of Technology, SE-100 44 Stockholm, Sweden \\ ${ }^{d}$ Tyndall National Institute, Lee Maltings Complex Dyke Parade, T12 R5CP Cork, Ireland \\ ${ }^{e}$ VLC Photonics S.L., Ed. 9B, D2, UPV, Camino de vera sn, 46022 Valencia, Spain \\ ${ }^{f}$ Commscope Connectivity Belgium, Diestsesteenweg 692, 3010 Kessel LO, Belgium \\ ${ }^{g}$ imec vzw. 3DSIP Department, Si Photonics Group, Kapeldreef 75, 3001 Leuven, Belgium \\ wim.bogaerts@ugent.be
}

\begin{abstract}
Programmable photonic circuits electronically reconfigure the flow of light on a chip. This requires hundreds or thousands of phase shifters and tunable couplers. Silicon photonic MEMS offer both high integration density and low power consumption. (C) 2020 The Author(s)
\end{abstract}

\section{Introduction}

Silicon Photonics makes it possible to integrate complex photonic circuits with thousands of components on a chip. This is leading us to programmable photonics: photonic circuits where light is controlled through electronics and software, using meshes of phase shifters and tunable couplers connected by waveguides [1]. Depending on their shape, these meshes can perform generic linear operations such as matrix-vector multiplications [2], single-value decomposition, or they can be configured as filter circuits for microwave signals [1].

In such generic circuits, the number of electro-optic actuators roughly scales with the square of the number of ports, rapidly growing into the hundreds or thousands. That means that compact and power-efficient tunable couplers and phase shifters are needed, as the current approach of waveguide heaters will not effectively scale up. In the European MORPHIC project, we develop an approach based on micro-electromechanical systems (MEMS), using electrostatic actuation to induce a phase shift in silicon waveguides, and combine this with the necessary electronics, packaging schemes and software algorithms to create a generic programmable photonic circuit.

\section{Silicon Photonic MEMS}

MEMS are an effective way of electrically changing the optical properties of a waveguide: by electrically moving material, a large change in refractive index can be induced [4]. However, integrating MEMS with optical waveguides requires that the waveguides become suspended. We developed a process to post-process the MEMS on the existing silicon photonics platform of IMEC, which already contains high-speed modulators and germanium photodetectors $[3,5]$. For this, the silicon waveguides are locally exposed and underetched using vapor-phase hydrofluoric acid. This results in compact phase shifters and tunable waveguide couplers [3,5].

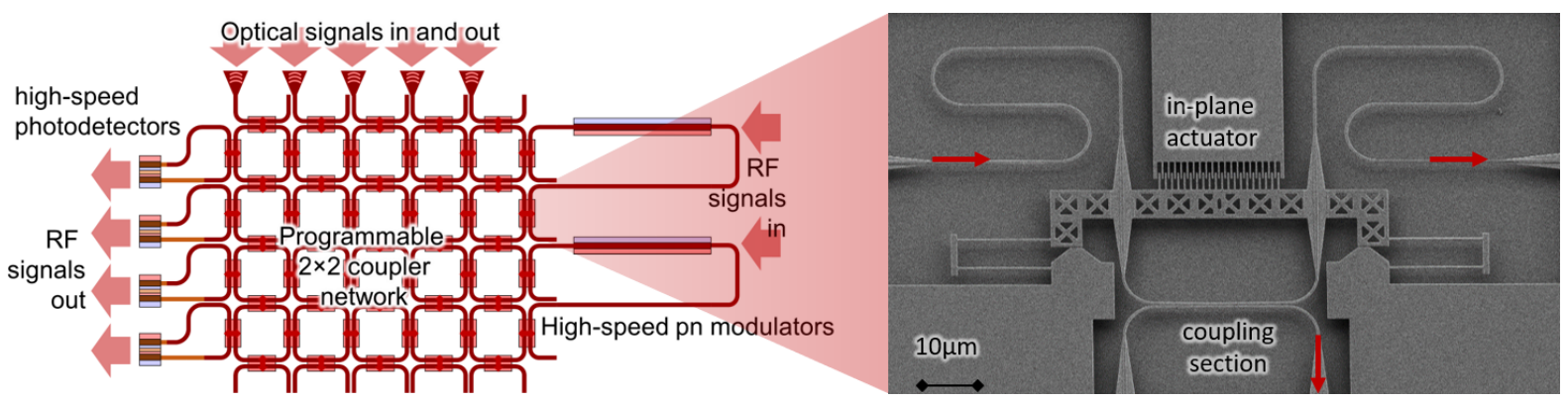

Fig. 1. A programmable PIC circuit schematic with a MEMS tunable coupler [3] 


\section{Scaling to larger circuits}

These MEMS actuators are combined into large-scale circuits, forming a waveguide mesh that can be electrically controlled. Different mesh topologies are possible, from forward-only meshes for linear computations, to generic recirculating meshes for programmable filters. Because the MEMS waveguides require transitions between suspended waveguides and oxide-clad waveguides, the choice of mesh topology is important to reduce excess transition losses and backreflections. Also, when scaling up to larger meshes, partitioning strategies become important to break up excessively long optical paths. These form a new class of optical placement and routing problems that require custom algorithms to design and program these new photonic circuits [6].

\section{Integration}

A programmable photonic circuit is more than the photonic chip. The many actuators need to be electrically controlled, which requires close integration with electronics. In the case of MEMS, this requires driving voltages in the range of 20-50V, which imposes additional constraints on the packaging. Also, some applications require highspeed microwave in/outputs, so it becomes clear that integration strategies based on flip-chipping, 3D stacking or 2.5D interposers are required. To protect the MEMS devices, which are sensitive e.g. to particles and humidity, a hermetic wafer-level sealing process is applied [7] before the connection with the electronics. To use a programmable circuit through the electronics stack, software control layers are needed, from simple feedback loops and parasitic control [8] to high-level programming schemes such as routing [6] or filter synthesis [1].

\section{Applications}

Programmable PICs can be deployed in two likely scenarios. The linear optical circuits are exceptionally useful for applications which require matrix algebra, such as artificial neural networks [2], optical qubit processing, or beamforming. This requires the integration of waveguide meshes in custom-designed application-specific PICs. However, programmable PICs can also be designed to perform a multitude of functions, limited only by the software-defined connectivity. The use-case, and the ecosystem for such general-purpose PICs, resembles that of electronic FPGAs [9]. Like FPGAs, they could be purchased off the shelf and programmed for a variety of scenarios, such as switching applications or microwave processing for sensing or wireless communication.

\section{Summary}

Large-scale programmable photonics presents an opportunity for silicon photonics to address a wide variety of applications. However, the technology stack for these new chips imposes significant requirements on the packaging, electronics and software algorithms. We develop a silicon photonics process with integrated MEMS to scale up to large circuits with lower power consumption.

\section{Acknowledgements}

This work was supported by the European Union grants 780283 (MORPHIC) and 725555 (PhotonicSWARM).

\section{References}

1. D. Pérez et al., "Programmable multifunctional integrated nanophotonics - Review Article," Nanophotonics 2018 7, 1351-1371 (2018).

2. N. C. Harris et al., "Linear programmable nanophotonic processors," Optica 5, 1623 (2018).

3. W. Bogaerts et al., "MORPHIC : Programmable photonic circuits enabled by silicon photonic MEMS," Proc. SPIE 11285, 11285-1 (2020).

4. C. Errando-Herranz et al., "MEMS for Photonic Integrated Circuits," IEEE J. Sel. Top. Quantum Electron. 26, 1-1 (2019).

5. N. Quack et al., "MEMS-enabled Silicon Photonic Integrated Devices and Circuits," IEEE J. Quantum Electron. PP, 1 (2019).

6. X. Chen et al., "ME2.2 - A Graph-based Design and Programming Strategy for Reconfigurable Photonic Circuits," 2019 IEEE Photonics Soc. Summer Top. Meet. Ser. (SUM) pp. 1-2 (2019).

7. X. Wang et al., "Wafer-level vacuum sealing by transfer bonding of silicon caps for small footprint and ultra-thin MEMS packages," J. Microelectromechanical Syst. 28, 460-471 (2019).

8. I. Zand et al., "Effects of Coupling and Phase Imperfections in Programmable Photonic Hexagonal Waveguide Meshes," Photonics Res. 8 (2019).

9. W. Bogaerts et al., "Programmable Photonics: An Opportunity for an Accessible Large-Volume PIC Ecosystem,” IEEE J. Sel. Top. Quantum Electron. 26 (20). 
OSA Advanced Photonics Congress

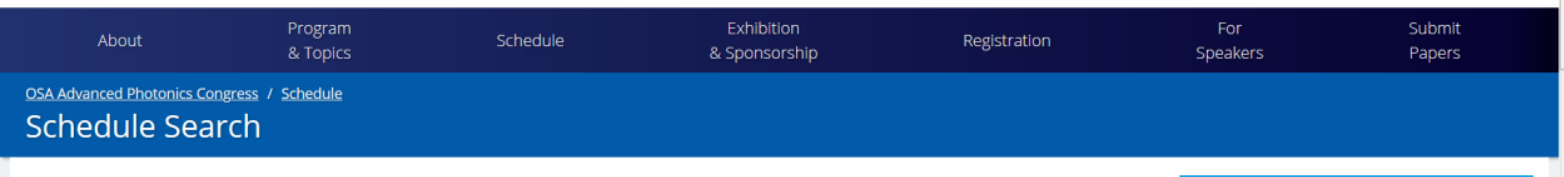

\section{Digital Forum
Schedule Search}

Search for

Attendee Help

bogaerts

Search mode:

Any word

$\cdot$

Search Filters

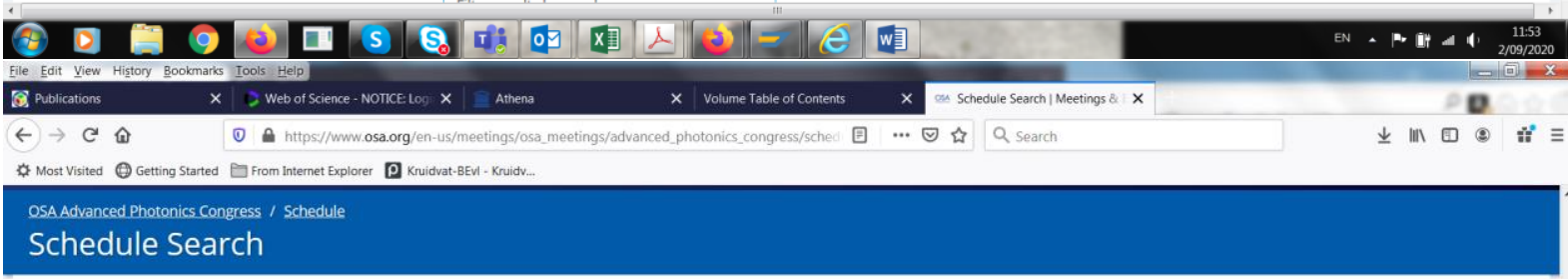

OSA Advanced Photonics Congress / Schedule

Schedule Search
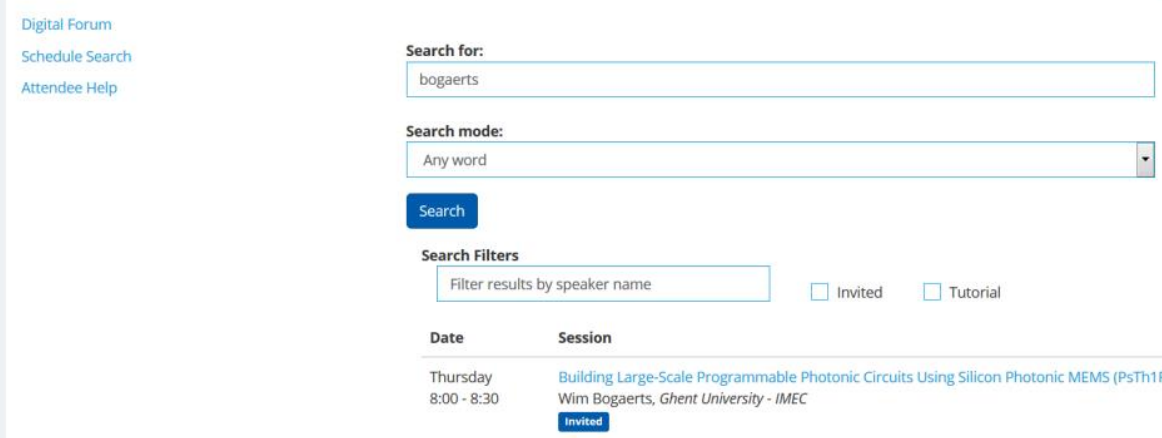

\section{Search}

Search Filters

Filter results by speaker name $\quad \square$ Invited $\quad \square$ Tutorial

Date Session

Thursday Builting Large-Scale Programmable Photonic Circuits Using Sillicon Photonic MEMS (PSTh1 E1)

8:00-8:30 Wim Bogaerts, Ghent University - IMEC

Invited 